\title{
Measurement of Acoustic Noise Effect Due to the Gradient Pulsing in functional Magnetic Resonance Imaging (fMRI)
}

\author{
SungTaek Chung ${ }^{1}$, InChan Song ${ }^{2}$, and HyunWook Park ${ }^{1}$ \\ 1. Dept. of Electrical Engineering, KAIST, 373-1 Kusung-dong, Yusung-gu, Taejon, Korea \\ 2. Institute of Radiation Medicine, Seoul National University College of Medicine, Seoul, Korea
}

\begin{abstract}
In MRI, gradient magnetic fields are used to obtain the spatial information by frequency modulation of the received signal. The gradient fields are generated by switching currents on the gradient coils, which generates acoustic noise due to Lorentzian force. In particular, fast imaging methods, which are usually used for fMRI, require fast switching of the gradient pulse, thereby generating large acoustic noise. The intensity of the acoustic noise depends on the imaging method and the pulse sequences. The acoustic noise induced by gradient pulsing may interfere for signal enhancement of brain areas with the presentation of auditory stimuli during fMRI. In this paper, the gradient pulsing effects on fMRI are analyzed for different combinations of gradients. The experimental results show that total activations by visual stimulation are slightly larger for a combination of $Z$ readout and $\mathrm{Y}$ phase-encoding gradients than those for a combination of $\mathrm{Y}$ readout and $\mathrm{Z}$ phase-encoding gradients when sagittal-view fMRI is performed.
\end{abstract}

Keywords: Acoustic Noise, functional MRI, Echo Planar Imaging (EPI), Visual Cortex

\section{INTRODUCTION}

Functional magnetic resonance imaging (fMRI) has been widely applied for imaging of the functional activation in human brain [1]. In most of these studies, the blood oxygenation level dependent (BOLD) or susceptibility contrast [2-7] can be exploited to provide activation maps when performing various tasks. Because deoxyhemoglobin is paramagnetic and oxyhemoglobin is diamagnetic, changes of the oxyhemoglobin / deoxyhemoglobin ratio induce changes of the local magnetic field homogeneity. That is, the local concentrations 
of deoxyhemoglobin within the brain lead to fractional changes of signal intensity in PMRI. However, the acoustic noise due to the gradient pulsing during MRI scanning can affect the accuracy of fMRI because the gradient pulsing noise causes patients to be discomfort as well as anxious (particularly for infants, children) during the MR imaging [8-10]. The acoustic noise due to gradient pulsing, which is generated by a Lorentzian force law, is an unavoidable phenomenon in MRI, and the MRI pulse sequences inherently produce the acoustic noise by causing rapid alternations of current in the gradient coils.

Only a few studies have been analyzed so far on the effects of acoustic noise for brain fMRI such as the visual cortex response [11]. When volunteers are exposed to a strong acoustic noise, the visual cortex response produces a smaller activation than in the case of relatively weak acoustic noise. Consequently, acoustic noise is one of the serious factors that obscure the tiny BOLD signal changes in fMRI of the brain. Because fast-imaging sequences, such as the echo-planar imaging (EPI) sequence [12], are used for fMRI and generate strong acoustic noise, the acoustic noise of gradient pulsing in fast imaging can degrade the performance of $\mathrm{fMRI}$. The acoustic noise generated by the gradient-echo EPI sequence measured an excess of $100 \mathrm{~dB}$ in the $\mathrm{C}$ mode from the GE 1.5-T scanner [13].

In this paper, the acoustic noise effects on fMRI are qualitatively analyzed for two different combinations of readout and phase-encoding gradients on sagittal-view images. Based on this analysis, an efficient gradient combination is suggested for sagittal view fMRI. Several experimental results are given to show the effect of gradient pulsing on fMRI.

\section{MATERIALS AND METHODS}

The gradient coils in the superconducting magnet system are subject to large Lorentzian forces because the current in the gradient coils is time-varying. The Lorentzian force, $\mathbf{F}$, acting on a conductor of length $\mathbf{L}$ carring a current $I$ and which resides in a magnetic field $\mathbf{B}$ is given by

$$
\mathbf{F}=\Pi \mathbf{L} \times \mathbf{B}=\mathbf{k} / L B \sin \alpha
$$

Where $\mathbf{L}=\mathrm{Li}, \mathbf{B}=\mathrm{Bj}$ and $\mathbf{F}=\mathrm{F} \mathbf{k}$ in which $\mathbf{i}, \mathbf{j}$, and $\mathbf{k}$ are the unit vectors of conductor, magnetic field and Lorentzian force directions, respectively, and $\alpha$ is the angle between $\mathbf{i}$ and $\mathbf{j}$. when $\alpha=90^{\circ}$, the line integral of $\mathbf{F}$ becomes zero around any closed current loop.

Among the three gradient coils for conventional MRI scanners, relatively large acoustic noise is generated by the $\mathrm{X}$ - and the Y-gradient coils, i.e., the transverse gradient coils. The Z-gradient coil set consists of two circular loops, and the magnetic field generated by one loop is in the opposite direction to the magnetic field generated by the other loop. Therefore, the acoustic noise in the Z-gradient coil is relatively small because the net Lorentzian force of the two circular loops is nearly zero $[9,14,15]$. Especially, for fast-imaging sequences 
such as echo-planar imaging (EPI), the most dominant noise source is the readout gradient which is a large and rapidly modulated gradient. For example, a sagittal-view image is obtained by using the selection gradient $\mathrm{X}$ and the combination of the readout and the phase-encoding gradients of $Z$ and $Y$, or $Y$ and $Z$. These two combinations of readout and phase-encoding gradients have different acoustic noise levels. Thus, we analyzed the signal changes due to gradient pulsing in functional MRI by using combinations both the $\mathrm{Y}$ readout $/ \mathrm{Z}$ phase-encoding gradients and the $\mathrm{Z}$ readout / $\mathrm{Y}$ phase-encoding gradients.

Experiments were performed using a standard clinical GE 1.5-T Signa MRI system with a quadrature head coil and a single-shot gradient-echo EPI pulse sequence. The pulse sequence employed an initial $\pi / 2$ pulse, an effective echo time of $60 \mathrm{~ms}$, and a repetition time of $3000 \mathrm{~ms}$. Other parameters included a field of view (FOV) of $24 \mathrm{~cm}$, a slice thickness of $5 \mathrm{~mm}$, and an image size of $128 \times 128$, which corresponded to voxel size of 1.875 $\mathrm{mm} \times 1.875 \mathrm{~mm} \times 5 \mathrm{~mm}$.

In the first experiment, the acoustic noise for the EPI pulse sequence was measured by using an acoustic level sensor in the imaging region. In sagittal-view EPI, the acoustic noise level of the $\mathrm{Y}$ readout $/ \mathrm{Z}$ phaseencoding gradients was about $104.5 \mathrm{~dB}$, whereas that of the $\mathrm{Z}$ readout / Y phase-encoding gradients was about $96.2 \mathrm{~dB}$. The second experiment was performed to verify the susceptibility effect on the signal intensity of fMRI when the readout and the phase-encoding gradients were changed. For this experiment, a phantom was designed to emphasize the susceptibility effect. The phantom consisted of big cylinder of water $(\phi: 15 \mathrm{~cm})$ in which three small cylinders were placed $(\phi: 5 \mathrm{~cm})$ as shown in Fig. 1 . Small air-filled tubes $(\phi: 1 \mathrm{~mm}, 3 \mathrm{~mm}, 4.5 \mathrm{~mm})$ were placed at the isocenters of the water-filled small cylinders.

Finally, a series of sagittal-view EPI images for functional MRI was obtained from healthy volunteers. Sixty-three sequential images for each volunteer were acquired with visual stimulation. In order to show the effect of gradient pulsing noise, these experiments were duplicated for $\mathrm{Y}$ readout / $\mathrm{Z}$ phase-encoding gradients and $\mathrm{Z}$ readout / $\mathrm{Y}$ phase-encoding gradients.

Visual stimulation was accomplished by a checkerboard screen of black and white rectangles that were alternated at a frequency of $8 \mathrm{~Hz}$. The first 7 images were obtained without visual stimulation, i.e., with a uniformly dark screen, whereas the next 7 images were obtained with visual stimulation of the checkerboard screen. This sequential paradigm without and with visual stimulation was repeated 4 times, with the last 7 images being obtained without visual stimulation.

We used the approach described by Bandettini et al. [16] to identify the activated fMRI pixels by a pixelby-pixel cross-correlation with the reference waveform of visual stimulation and by thresholding the crosscorrelation coefficients with a threshold value of 0.4 . Considering the number of images included in the crosscorrelation analysis, this gave an effective $P$ value $<0.0015$. In order to compare the activations from the two combinations of readout / phase-encoding gradients, the number of activated pixels and the average intensities of the activated pixels were analyzed, where the total activation is defined by multiplying the number of activated pixels by the average intensities of the activated pixels [11]. 




Figure 1. A phantom containing water and air to show the susceptibility effect with respect to the readout and the phase-encoding gradients.

\section{RESULTS}

Figure 2 shows the sagittal-view phantom images which were obtained with (a) $Z$ readout / Y phaseencoding gradients and (b) $\mathrm{Y}$ readout $/ \mathrm{Z}$ phase-encoding gradients. As shown in Fig. 2, a spearhead-shaped deformation is generated by the magnetic susceptibility effect around the air-filled tubes and depends on the orientation of the readout gradient $[17,18]$. In order to verify the signal change due to changing the readout / phase-encoding gradients, the same regions were selected from the two images in Fig. 2. Regions 1, 2, and 3 were selected to analyze the intensity variation around the spearhead-shaped deformations, whereas region 4 was selected to analyze the intensity variation in uniform area. The sizes of regions $1,2,3$, and 4 were $4.2,3.3,1.6$, and $3.3 \mathrm{~cm}^{2}$, respectively. A summary of the signal variations for the two combinations of readout / phaseencoding gradients is given in Table 1. From Table 1, it is found that there was a little change in the signal intensity between the two different combinations of readout / phase-encoding gradients for sagittal-view images, although a spearhead-shaped deformation appeared along the direction of the readout gradient. The susceptibility effect for human body imaging is usually less than that of this phantom imaging. Therefore, this phantom study shows that the signal change due to different gradient combinations is negligible.

Figure 3 shows the distributions of activated pixels in the visual cortex region. The five images in Fig. 3a were obtained with the $\mathrm{Y}$ readout $/ \mathrm{Z}$ phase-encoding gradients whereas the other five images in Fig. $3 \mathrm{~b}$ were obtained with the $\mathrm{Z}$ readout / Y phase-encoding gradients. The numbers of activated pixels, the average intensities, and the total activation for each of five volunteers are given for both gradient combinations in Table 2. The number of activated pixels in the case of a strong acoustic noise environment ( $\mathrm{Y}$ readout $/ \mathrm{Z}$ phaseencoding) is slightly smaller than that in a weak acoustic noise environment ( $Z$ readout / $Y$ phase-encoding). In order to show the difference in the activation between the two combinations of readout / phase-encoding gradients, the signal variations for the case of the two gradient combinations are shown in Fig. 4, which is 
derived from total activation for all volunteers. The periods of visual stimulation are described by the solid lines in Fig.4.

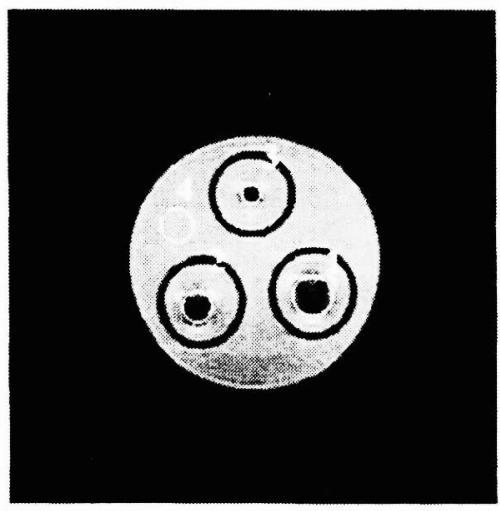

a

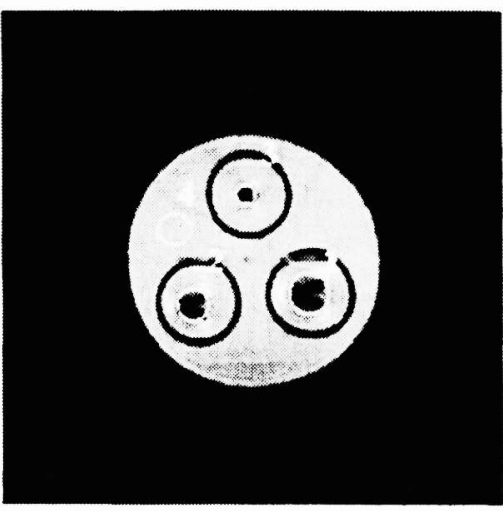

b

Figure 2. Sagittal view phantom images (a) with $Z$ readout / Y phase-encoding and (b) with $Y$ readout $/ Z$ phase-encoding. The circles 1, 2, 3, and 4 were selected for analysis of signal deformation.

Table 1. Summary of intensity data for the phantom images.

\begin{tabular}{|c|c|c|c|c|c|}
\hline \multirow{2}{*}{ Regions } & \multicolumn{2}{|c|}{ Areas $\left(\mathrm{cm}^{2}\right)$} & \multicolumn{2}{|c|}{ Average pixel intensity } & \multirow{2}{*}{ Ratio** } \\
\hline & $R D(Z) / P E(Y)^{*}$ & $\mathrm{RD}(\mathrm{Y}) / \mathrm{PE}(\mathrm{Z})$ & $R D(Z) / P E(Y)$ & $R D(Y) / P E(Z)$ & \\
\hline 1 & 4.2 & 4.2 & 920.3 & 900.6 & 1.022 \\
\hline 2 & 3.3 & 3.3 & 1032.9 & 1011.0 & 1.022 \\
\hline 3 & 1.6 & 1.6 & 1213.6 & 1211.9 & 1.002 \\
\hline 4 & 3.3 & 3.3 & 2028.2 & 2026.0 & 1.001 \\
\hline Mean \pm SD & & & & & $.012+0.024$ \\
\hline
\end{tabular}

* Readout Gradient (RD) / Phase-Encoding (PE) Gradient

** The ratio for the average pixel intensity shows little intensity variation during the $R D(Z)$ / $P E(Y)$ sequence and during the $R D(Y)$ / $P E(Z)$ sequence. 


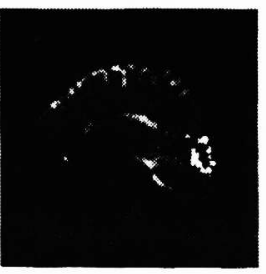

\#1

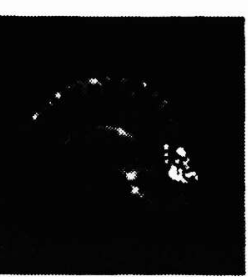

\#1

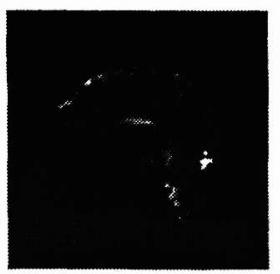

\#2



\#2

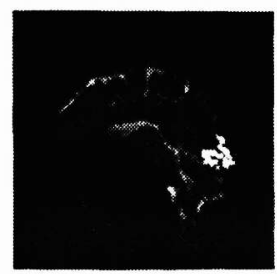

\#3

a

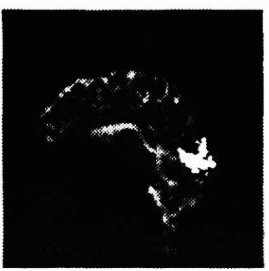

\#3



\#4

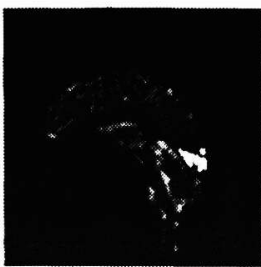

\#4

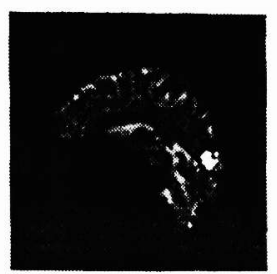

\#5

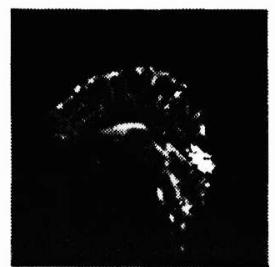

\#5

Figure 3. Comparison of total activations by acoustic stimulation obtained by using different combinations of readout and phase-encoding gradients for multi-slice sagittal-view images: (a) $\mathrm{Y}$ readout $/ \mathrm{Z}$ phase-encoding gradients and (b) $\mathrm{Z}$ readout / $\mathrm{Y}$ phaseencoding gradients.

Table 2. Summary of the total activations from the two combinations of readout and phaseencoding gradients for sagittal-view images.

\begin{tabular}{|c|c|c|c|c|c|c|c|}
\hline \multirow{2}{*}{ Volunteers } & \multicolumn{2}{|c|}{$\begin{array}{l}\text { No. of activated pixels } \\
\text { according to image plane axis }\end{array}$} & \multicolumn{2}{|c|}{$\begin{array}{c}\text { Average } \\
\text { pixel intensities }\end{array}$} & \multicolumn{2}{|c|}{ Total activation $\left(A_{1}\right)$} & \multirow{2}{*}{ Ratio } \\
\hline & $\mathrm{RD}(\mathrm{Z}) / \mathrm{PE}(\mathrm{Y})$ & $R D(Y) / P E(Z)$ & $R D(Z) / P E(Y)$ & $R D(Y) / P E(Z)$ & $R D(Z) / P E(Y)$ & $R D(Y) / P E(Z)$ & \\
\hline A & 360 & 262 & 1.0360 & 1.0398 & 373 & 272 & 1.371 \\
\hline B & 376 & 354 & 1.0354 & 1.0328 & 389 & 366 & 1.063 \\
\hline C & 344 & 297 & 1.0315 & 1.0299 & 355 & 306 & 1.160 \\
\hline$D$ & 361 & 243 & 1.0180 & 1.0230 & 368 & 249 & 1.478 \\
\hline$E$ & 347 & 252 & 1.0358 & 1.0311 & 359 & 260 & 1.381 \\
\hline Mean $\pm S D$ & $358 \pm 11.4$ & $282 \pm 40.6$ & $1.0350 \pm 0.008$ & $1.0313 \pm 0.005$ & $369+8.4$ & $291 \pm 42.3$ & $.291 \pm 0.154$ \\
\hline
\end{tabular}

* Readout Gradient (RD) / Phase-Encoding (PE) Gradient

" The ratio** for total activation was larger during the $Z$ (readout) / $Y$ (phase-encoding) sequence than during the $Y$ (readout) / $Z$ (phase-encoding) sequence. 


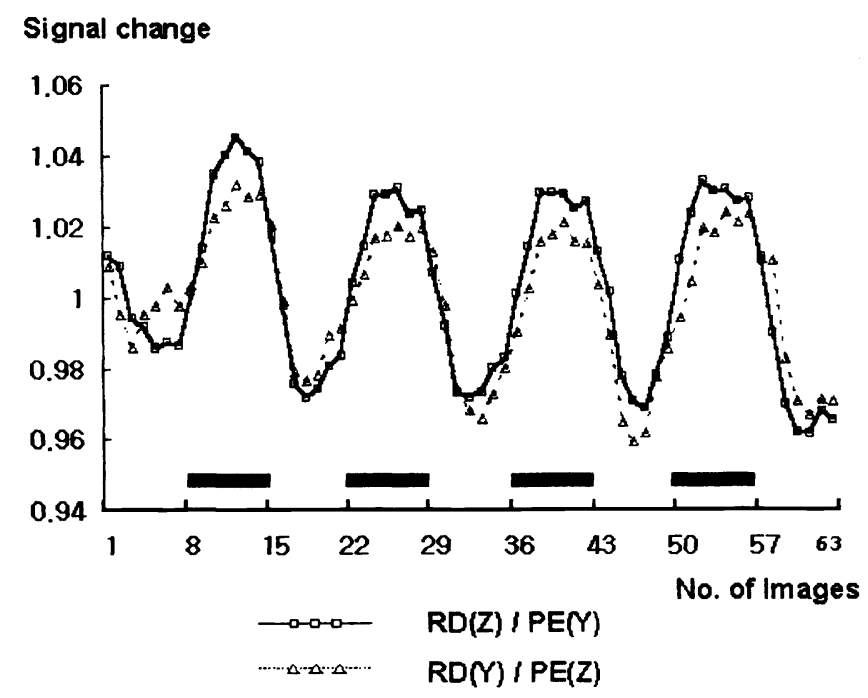

Figure 4. Comparison of the signal changes from all activated pixels for the five volunteers.

\section{DISCUSSION AND CONCLUSIONS}

In the experiment, we observed an effect of acoustic noise on brain fMRI. Visual response has many activations when subjects are exposed to a weak acoustic noise of $Y$ phase encoding / $Z$ readout gradient compared with relatively strong acoustic noise of $\mathrm{Y}$ readout $/ \mathrm{Z}$ phase encoding gradient. The average total activation signal is increased about $27 \%$ for the combination of $Z$ readout / Y phase-encoding gradients in comparison with the combination of $\mathrm{Y}$ readout / $\mathrm{Z}$ phase-encoding gradients. This experiment result represents that visual response can be affected by acoustic noise. Therefore, the gradient combination of $Y$ phase encoding / $\mathrm{Z}$ readout is efficient for sagittal view fMRI since the combination has less acoustic noise.

In conclusion, functional MRI should be performed with utmost reduction of acoustic noise due to gradient pulsing. Small step of improvement can be obtained by using sagittal-view imaging and a $Z$ readout / Y phase-encoding gradient combination. This is an important step for patients undergoing MRI as well as for those undergoing functional MRI.

\section{REFERENCES}

1. Belliveau JW, Kennedy DN, McKinstry RC, et al., "Functional mapping of the human visual cortex by magnetic resonance imaging," Science 254, pp. 716-719, 1991.

2. Ogawa S, Tank DW, Menon R, et al., "Intrinsic signal changes accompanying sensory stimulation: functional brain mapping with magnetic resonance imaging," Proc Natl Acad Sci USA 89, pp. 
5951-5955,1992.

3. Kwong KK, Belliveau JW, Chesler DA, et al., "Dynamic magnetic resonance imaging of human brain activity during primary sensory stimulation," Proc Natl Acad Sci USA 89, pp. 5675-5679, 1992.

4. Cho ZH, Ro YM, Park SH, Chung SC., "NMR functional imaging using a tailored RF gradient echo sequence: a true susceptibility measurement technique," Magn Reson Med 35, pp. 1-5, 1996.

5. Frahm J, Bruhn H, Merboldt KD., "Dynamic MRI of human brain oxygenation during rest and photic stimulation," J Magn Reson Imaging 2, pp. 501-505, 1992.

6. Ogawa S, Lee T-M., "Magnetic resonance imaging of blood vessels at high fields: in vivo and in vitro measurements and image simulation," Magn Reson Med 16, pp. 9-18, 1990.

7. Kim SG, Ashe J, Hendrich $\mathrm{K}$, et al., "Functional magnetic resonance imaging of motor cortex: hemispheric asymmetry and handedness," Science 261, pp. 615-617, 1993.

8. Brummett RE, Talbot JM, Charuhas P., "Potential hearing loss resulting from MR imaging," Radiology 169, pp. 539-540, 1988.

9. Hurwitz R, Lane SR, Bell RA, Brant-Zawadzki MN., "Acoustic analysis of gradient -coil noise in MR imaging," Radiology 173, pp. 545-548, 1989.

10. Quirk ME, Letendre AJ, Ciottone RA., "Anxiety in patients undergoing MR imaging," Radiology 170, pp. 463-466, 1989.

11. Cho ZH, Chung SC, Lim DW, Wong EK., "Effect of the gradient systems on fMRI: a study on auditory, motor, and visual cortices," Magn Reson Med 39, pp. 331-336, 1998.

12. Mansfield P., "Multi-planar image formation using NMR spin echoes," J Phys C 10, pp. L55-L58, 1977.

13. Cho ZH, Park SH, Kim JH, et al., "Analysis of acoustic noise in MRI," Magn Reson Imaging 15, pp. 815-822, 1998.

14. Cho ZH, Chung ST, Chung JY, et al., "A new silent magnetic resonance imaging using a rotating DC gradient," Magn Reson Med 39, pp. 317-321, 1998.

15. Mansfield P, Chapman BLW, Bowtell R, Glover P, Coxon R, Harvey PR., "Active acoustic screening: reduction of noise in gradient coils by Lorentz force balancing," Magn Reson Med 33, pp. 276-281, 1995.

16. Bandettini PA, Jesmanowicz A, Wong EC, Hyde JS., "Processing strategies for time-course data sets in functional MRI of the human brain," Magn Reson Med 30, pp. 161-173, 1993.

17. Ludeke KM, Roschmann P, Tischler R., "Susceptibility artefacts in NMR imaging," Magn Reson Imaging 3, pp. 329-343, 1985.

18. Park HW, Ro YM, Cho ZH., "Measurement of the magnetic susceptibility effect in high-field NMR imaging," Phys Med Biol 33, pp. 339-349, 1988. 